Inform. Stor. Retr. Vol. 4, pp. 333-341. Pergamon Press 1969. Printed in Great Britain

\title{
REMOTE ACCESS TO SCHEMATIC DRAWINGS
}

\author{
P. BRUCE BERRA \\ Purdue Laboratory for Applied Industrial Control, Purdue University, Lafayette, Indiana \\ DeAN H. WILSON \\ Department of Industrial Engineering, The University of Michigan, Ann Arbor, Michigan
}

\begin{abstract}
Summary-An electronic information system for storing, transmitting, receiving and displaying electrical circuit schematics is presented. The schematics are binary coded for computer storage and for transmission over telephone grade communications lines. A complete schematic can be transmitted in a matter of a few seconds with no degradation in the image quality. A standard set of schematic symbols and the associated alphanumeric characters are stored at the receiver in the character matrix of a shaped beam tube. Only the information as to the location of each schematic symbol in the character matrix and the organization of the schematic symbols is stored and transmitted.
\end{abstract}

\section{INTRODUCTION}

THE PAST few years have witnessed an extremely rapid expansion of the computer field with a resulting requirement for the display of information on visual devices. Significant advances have been made in the display of alphanumeric information; however, only recently has increased emphasis been directed toward the display of non-alphanumeric information. As a result, the engineer faces a situation which will involve him in automatic methods of information storage and retrieval. The development of mass storages and visual input/output devices with the capability of storing and retrieving pictorial as well as alphanumeric information will permeate many of the industrial operations with which he is concerned. This article presents a conceptual design of an electronic information system for the storing of binary coded electrical circuit schematics, the transmission of the codes, and the ultimate display of the complete schematic on the face of a shaped beam cathode ray tube.

\section{SYSTEM DESCRIPTION}

The information system consists of two main divisions, a central location and several satellite locations. The central location includes a central processing unit, an auxiliary storage unit, the requisite input/output devices and a buffer storage unit which is connected to a commercial telephone system. Each of the satellite locations includes a control console, a main shaped beam tube, an auxiliary shaped beam tube and a buffer storage unit which is connected to the central location via the telephone system. Figure 1 is a block diagram of the information system.

The library of schematics is stored in the auxiliary storage unit of the central location. If a schematic is desired by any one of the satellite locations, a directory of schematic numbers would be consulted. From this directory the number of the desired schematic would be obtained and keyed into the key unit on the control console. The number would then be binary coded and sent via telephone lines to the input/output unit of the central location, where it would be multiplexed into the central processing unit. Utilizing this number as the block address, the central processing unit would locate in the auxiliary storage unit the codes 


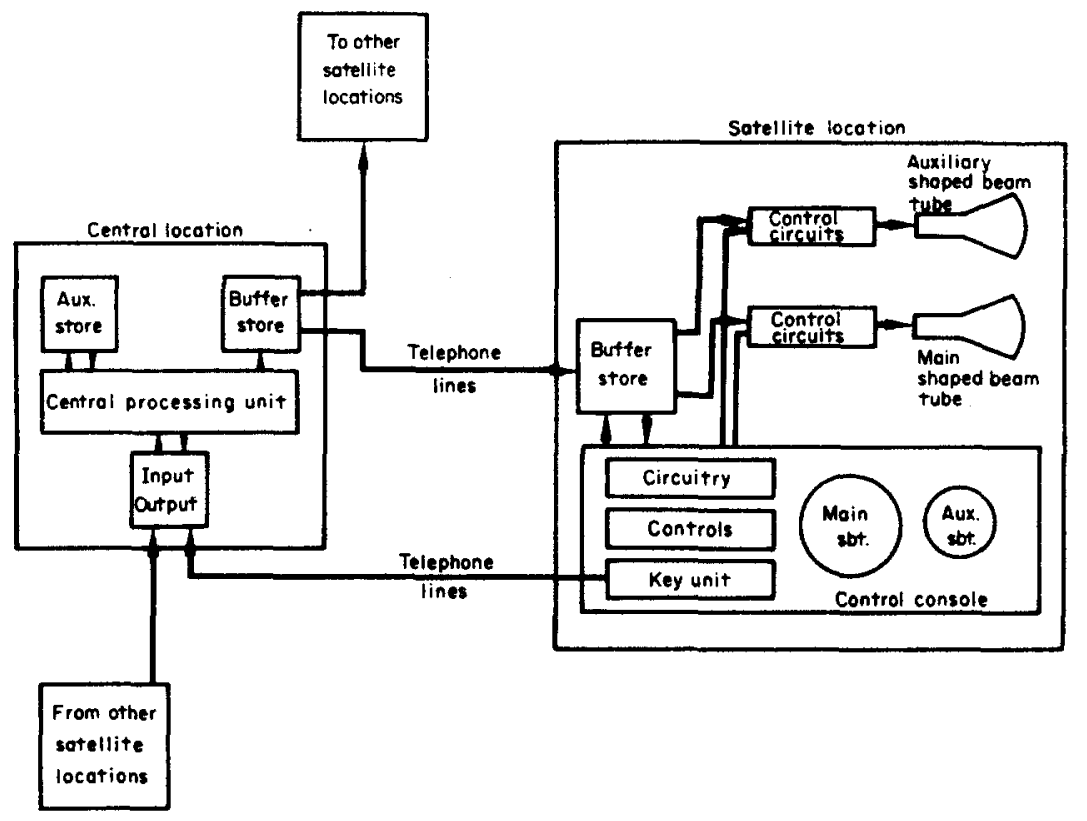

Fig. 1. Block diagram of the information system.

for the desired schematic. These codes would be placed in the buffer storage unit of the central location for transmission via telephone lines to the buffer storage unit of the requesting satellite location. These codes as well as codes generated at the satellite location would serve as input to the control circuits of the main shaped beam tube.

As the character matrix in a shaped beam cathode ray tube can contain, as part of the character set, the circuit schematic symbols, it is not necessary to transmit these circuit symbols from the central to the satellite location. Instead, it is necessary to transmit only the positions of the symbols in the matrix and the order in which these are to be displayed to produce the particular schematic. Because the number of different types of symbols on a schematic is small, fewer bits of information are required to choose it from the matrix than to code and transmit the symbol itself.

If further information regarding a particular component on the schematic is desired, the number of that component and the schematic number would be keyed into the control console. This information would be utilized in locating the desired information in the auxiliary storage unit at the central location. The information would then be transmitted to the satellite location and displayed on the auxiliary shaped beam tube. If a "hard copy" of the schematic or the component information is desired, the scope face could be photographed, or the information could be printed out on an auxiliary printer.

\section{CODING SYSTEM}

The coding and decoding of schematics for the information system can best be developed by examining in detail the generation of a schematic on the satellite location main shaped beam tube. Imagine a cartesian coordinate system superimposed on the face of the tube with the $(0,0)$ position in the upper left corner. Assume that there are 256 addressable locations in $X$ and 256 in $Y$. Then there are a total of 65,536 addressable scope face locations. These 
locations will be called nodes. Contained in the 65,536 addressable nodes are 4096 scope face locations which will be called major nodes. Thus there is a course grid superimposed on a fine grid.

Circuit symbols are placed only at the major nodes. They are expanded and centered about one major node with their terminals connecting to adjacent major nodes. They are expanded in order to preserve a proportion to alphanumerics that is in keeping with the proportion found on typical schematics.

Alphanumerics, which can be placed at any of the 65,536 scope face locations, are of two types: those associated with specific circuit symbols and those not necessarily associated with circuit symbols but which are required on the schematic. The former type is placed at a scope face location adjacent to the circuit symbol it describes and the latter type is placed on the scope face wherever it is required.

Sixteen bit, ten bit, and three bit codes are received from the central location. A sixteen bit code is a scope face location code. A ten bit code is divided into two parts, an eight bit code to locate one of the 200 circuit symbols and alphanumeric characters on the character matrix of the shaped beam tube and a two bit code which gives an indication of the next operation. The three bit code indicates how far to draw a line or how many nodes to move to the right.

The codes received from the central location are ordered with a sixteen bit code always being received first. This sixteen bit code locates a single node on the face of the tube. The nodes above and to the left of this node are the nodes which are used for the generation of the schematic. Thus, this code determines the size of the schematic that is being received. Through a simple linear transformation the schematic is centered on the face of the scope.

Beginning with the upper left major node in the block of nodes, and scanning across the nodes similar to typing a letter, the ordered codes produce circuit symbols and alphanumerics which are placed on and around the major nodes thus producing a circuit diagram. After all the major nodes have been scanned, the remaining alphanumerics are placed on the schematic.

\section{EXAMPLE OF CODING SYSTEM}

The simple circuit shown below will be used to illustrate the facilities of the coding system.

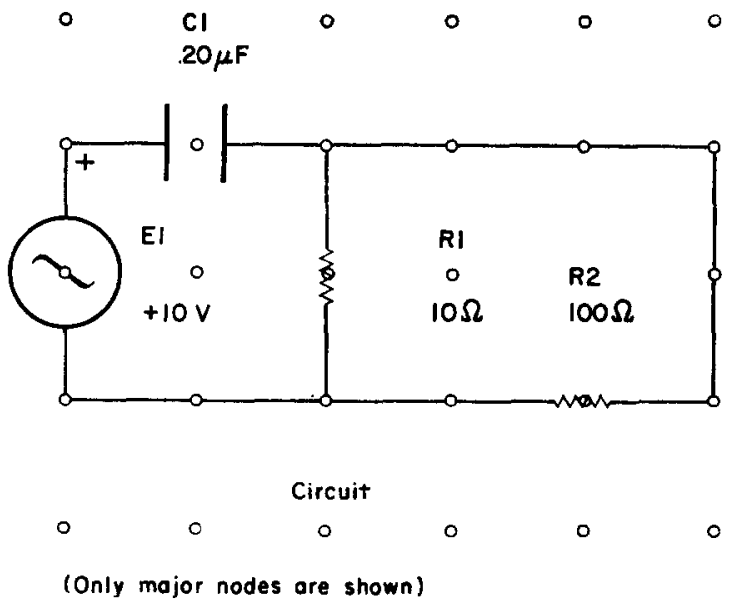


The sixteen bit scope face location code has eight bits for locating nodes in the $X$ and eight bits for locating nodes in the $Y$ direction. In this example the symbol $\{x, y\}$ will be utilized to signify a scope face location code. The code for a character in the character matrix will be given by the symbol $(a, b)$. The first symbol $(a)$ is for the eight bit code which locates the character in the character matrix and the second symbol $(b)$ is for the two bit code which indicates the next operation. The three bit code which indicates how far to draw a line or how many nodes to move to the right will be given by the symbol $\langle c\rangle$.

Suppose for this example that the characters in the character matrix are numbered as follows:

$\begin{array}{ll}1-1 & 21-\mathrm{C} \\ 2-2 & 22-\mathrm{D} \\ 3-3 & 23-\mathrm{E} \\ 4-4 & 24-\mathrm{F} \\ 5-5 & 25-\mathrm{G} \\ 6-6 & 26-\mathrm{H} \\ 7-7 & 27-\mathrm{I} \\ 8-8 & 28-\mathrm{J} \\ 9-9 & 29-\mathrm{K} \\ 10-0 & 30-\mathrm{L} \\ 11-\text { N } & 31-\mathrm{M} \\ 12-\xi & 32-\mathrm{N} \\ 13--1 \vdash & 33-\mathrm{O} \\ 14-\mathrm{Blank} & 34-\mathrm{P} \\ 15-\mathrm{Horizontal} \text { line } & 35-\mathrm{Q} \\ 16-\text { Vertical line } & 36-\mathrm{R} \\ 17-+ & 37-\mathrm{S} \\ 18-\Xi & 38-\mathrm{T} \\ 19-\mathrm{A} & 39-\mathrm{U} \\ 20-\mathrm{B} & 40-\mathrm{V} \\ & \text { etc. }\end{array}$

and the two bit code $(b)$ has the following meaning:

$b=0$ Alphanumeric information will be received next.

$b=1$ Skip one major node and place what is received next at the next major node.

$b=2$ Remain at that major node. The next information received will be a three bit message which will indicate how many nodes to move to the right or how far to draw a line.

$b=3$ Carriage return. That is all the information for this row of major nodes, go to the first major node in the next lower row and place the next piece of information there.

The codes received from the central location for the simple circuit would be as follows:

$\{21,9\}(14,2)\langle 1\rangle(13,0)(2,0)(10,2)\langle 1\rangle(15,2)\langle 3\rangle(16,2)\langle 2\rangle(14,3)(18,0)(17,0)$

$(1,0)(10,1)(12,0)(1,0)(10,3)(15,2)\langle 3\rangle(14,2)\langle 1\rangle(11,0)(1,0)(10,0)(10,3)\{7,12\}$

$2(1,0)(27,0)(36,0)(21,0)(39,0)(27,0)(38,1)\{2,2\}(17,2)$

An explanation of these codes follows:

$\{21,9\}$ - This code locates the node that is twenty-one nodes to the right and nine nodes down from the node in the upper left corner of the scope. This could be written 
equivalently as $\{6,3\}$ in terms of major nodes. The schematic will be placed at the nodes above and to the left of this node. Only alphanumerics associated with this schematic can be written outside this block of nodes. With a linear transformation the block of nodes is transformed to the center of the scope face.

$(14,2)$ - A blank is placed at the first major node and the 2 indicates that information as to how many nodes to move to the right will come next.

$\langle 1\rangle-$ Move one major node to the right and place what is received next at that node.

$(13,0)$ - Place a capacitor symbol at that node.

Alphanumeric information will come next.

Alphanumeric information is placed above a horizontally oriented circuit symbol and to the right of a vertically oriented circuit symbol. With the horizontal circuit symbol the first alphanumeric, $\mathrm{C}$ in this case, is placed at the major node above the symbol. The next alphanumeric is placed at the major node above the symbol. The next alphanumeric is placed at the next node to the right, the 1 in this case. The next row of alphanumerics for that symbol begins two nodes above the major node at which the circuit symbol is placed, 2 in this case, and subsequent alphanumerics are placed to the right of it. For the vertical circuit symbols, the first row of alphanumerics begins three nodes to the right and one up. The second row begins three nodes to the right and one down.

Codes for redundant alphanumerics, which are associated with circuit symbols, are not stored at the central location but are generated at the satellite location. In the case of the capacitor the alphanumerics, $C, 1, \mu$ and $F$ are generated at the satellite location. The code for capacitor $(13,0)$ is recognized by the system and the $C 1$ is placed in the proper location. After all the alphanumeric information for the capacitor is received, the alphanumerics $\mu F$ are placed on the scope. The next capacitor whether it be horizontal or vertical will be called $C 2$.

$(2,0)$-Place the 2 at its proper location. The next information received will be an alphanumeric.

$(10,2)-P l a c e$ the 0 at its proper location. The next information will indicate how many major nodes to move to the right.

$\langle 1\rangle$-Move one major node to the right and place what is received next at that node.

$(15,2)$-Draw a horizontal line. The next information received will indicate the length of the line.

<3) -Draw the line to the third node to the right.

$(16,2)$-Draw a vertical line. The next information received will indicate the length of the line.

$\langle 2\rangle$-Draw the line down two nodes and return to the original node.

$(14,3)$-Blank. That is all the information for that row of nodes. Go to the first major node in the next lower row and place what is received next at that node.

$(18,0)$-Place a source symbol at that node and place E1 at the proper nodes. Alphanumeric information will come next.

$(17,0)$ - Place a + at the proper node. Alphanumeric information will come next.

$(1,0)$-Place a 1 at the proper node. Alphanumeric information will come next. 
$(10,1)$ - Place a 0 at the proper node. Skip one major node and place what is received next at the next major node.

$(12,0)$ - Place a resistor symbol at that node. Alphanumeric information will come next.

$(1,0)$-Place a 1 at the proper node. Alphanumeric information will come next.

$(10,3)$-Place a 0 at the proper node. That is all the information for that row of nodes. Go to the first major node in the next lower row and place what is received next at that node.

$(15,2)$-Draw a horizontal line. The next information received will indicate the length of the line.

<3 -Draw the line to the third node to the right.

$(14,2)-$ Blank. The next information received will indicate how many nodes to move to the right.

$\langle 1\rangle-$ Move one node to the right and place what comes next at that node.

$(11,0)$ - Place a resistor symbol at that node. Alphanumeric information will come next.

$(1,0)$-Place a 1 at the proper node. Alphanumeric information will come next.

$(10,0)$-Place a 0 at the proper node. Alphanumeric information will come next.

$(10,3)$ - Place a 0 at the proper node. That is all the information for that row of nodes. Go to the first major node in the next lower row and place what is received next at that node.

When this code is received for the last major node, it indicates that all the schematic information has been received except special alphanumerics. The next information to be received will be a scope face location.

$\{7,12\}$ - Place the next alphanumeric at this location.

$(21,0)(27,0)(36,0)(21,0)(39,0)(27,0)$-These codes are used to write the letters CIRCUI in a row.

$(38,1)$-Place a $\mathrm{T}$ at the proper node. The next information received will be a location.

$\{2,2\}$-Place the next alphanumeric at this location.

$(17,3)$-Place $a+$ at the proper node and stop.

Note that after the last major node utilized in reproducing the schematic was reached, the $(, b)$ code took on a somewhat different meaning.

$b=0$ Alphanumeric information will come next.

$b=1$ A location code will come next.

$b=2$ Stop. End of information.

$b=3$ Blank. Not used.

If there is no alphanumeric information after the last major node the location of that node is sent followed by a blank with the stop code.

Four circuits ranging from a simple RLC circuit to a fairly complex strain gage signal amplifier circuit were coded by the above method and the total number of transmitted bits determined. A plot of the number of bits vs. the number of alphanumerics and circuit symbols is shown in Fig. 2 for the four circuits. 


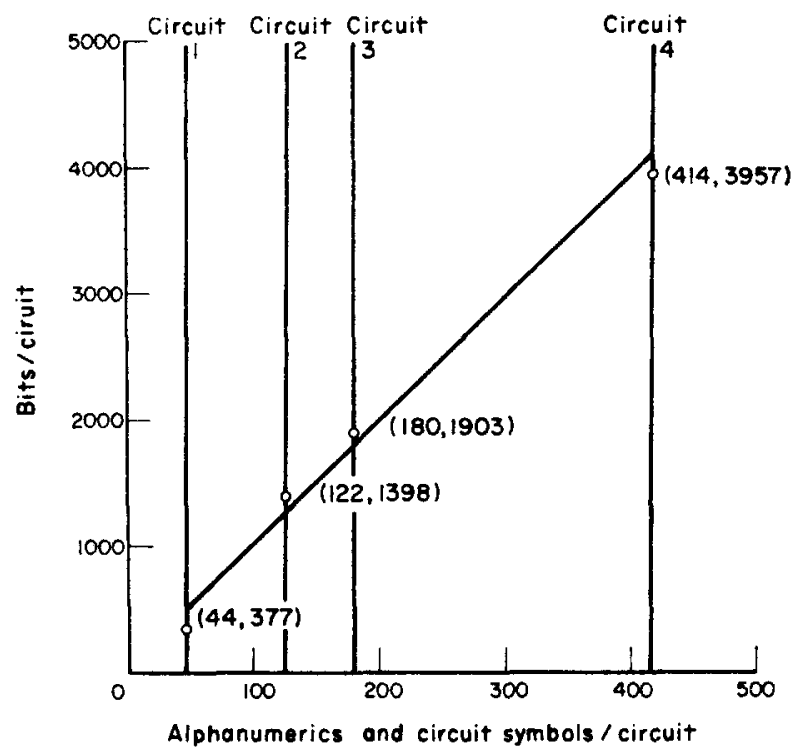

FIG. 2. Bits per circuit vs. alphanumerics and circuit symbols per circuit.

Only the above system of coding is presented here while in fact several systems and modifications thereof were considered. Each offers certain advantages and disadvantages. Advantages in that the total number of bits transmitted over telephone lines can be reduced and disadvantages in that the satellite equipment tends to become more complex and probably more expensive. An interesting problem for further investigation would be to determine the most efficient method of coding a schematic. In developing the method the following observations could be utilized to advantage: (1) A large number of circuit diagrams have only a few different types of symbols. (2) The symbols may be repeated several times in the schematic. (3) Entire sections of a schematic may be repeated several times.

\section{COST DETERMINATION}

The total equipment and installation cost for five satellite locations and one central location utilizing presently available equipment would be about $\$ 1,224,500$ and the total operation, maintenance and rental cost would be about $\$ 160,000$ per year. Assuming a rate of return on investment of 5 per cent, an estimated life for the equipment of $7 \mathrm{yr}$, the annual cost of capital recovery for the initial investment is $\$ 180,452$. This value, taken with the yearly disbursements, yields a total equivalent annual cost of $\$ 340,452$ for the estimated life of the equipment.

One of the four circuits was taken as a typical circuit in the calculation of the cost of obtaining a schematic. It contained about 2000 bits and therefore could be transmitted across the transmission link in approximately one second. If the five satellite locations share the cost of the system equally, then each would be assessed $\$ 68,090$ annually or $\$ 186.50$ daily. Therefore, if an average cost of one dollar for the typical circuit is desired, the system must be in continuous operation for only a little over three min each day. Figure 3 depicts the relationship between the cost of obtaining the circuit in cents vs. the minutes of continuous transmission per day. 


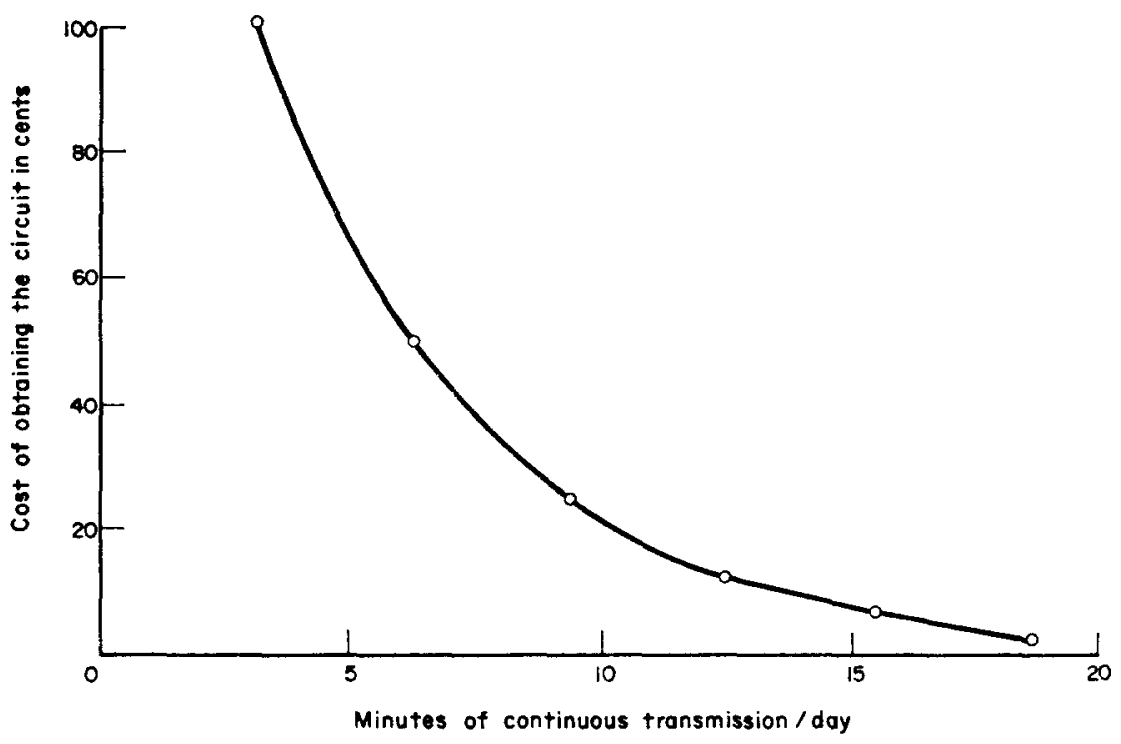

FIG. 3. The cost of obtaining the circuit vs. the minutes of continuous transmission per day.

Further savings can be obtained if the computer at the central location is utilized for other purposes when it is not performing functions for the information system. At the satellite locations the buffer store and the display equipment can also be utilized for other purposes when not required for obtaining schematics.

\section{SYSTEM ADVANTAGES}

The information system has the following advantages:

(1) The coding scheme for the schematics takes advantage of the fact that much of the information required for the display of a schematic is already at the satellite location. Therefore, only information that is absolutely necessary is transmitted from the central location.

(2) The schematic, as observed by the operator on the shaped beam tube is generated at the satellite location and thus no degradation of image quality is observed because of transmission line quality.

(3) Only one master set of schematics is required and these schematics are always at the central location in computer storage.

(4) Any modifications of the schematic that must be made are made only on the master set of schematics and the modified information is transmitted to those satellite locations that have requested those particular schematics in the past.

(5) The cost of the system is well within the budget of most medium sized industrial organizations.

(6) Only the schematics that are of interest to a satellite location need be requested from the central location. This greatly reduces the amount of unnecessary information at the satellite location.

(7) An off-center/expand mode allows the operator to view in expanded form any portion of the schematic. 
Acknowledgments-This work was undertaken at The University of Michigan by Mr. Berra as partial fulfilment of the requirements for a graduate degree in Industrial Engineering. Professor Wilson was responsible for guiding the research.

\section{REFERENCES}

[1] RoRert W. Johnson: Digital data display systems, An assessment. Computers and Automation, May, 1964.

[2] M. David PrInce: Man-computer graphics for computer-aided design. Proc. I.E.E.E., 1966, 54, 1698.

[3] J. R. ShanNaRd: High speed communications of graphic intelligence with hard copy readout. Am. Inst. elect. Engrs Trans., 1962, 81, 1.

[4] R. A. Siders: Computer Graphics. American Management Association, New York, New York, 1966.

[5] I. E. SuTHERLAND: Sketchpad: A man-machine graphic communication system, Proc. Spring Joint Computer Conf., 1963, 22, 329. 